\title{
Querelles historiennes sur la Révolution française : l'argumentation par le chiffre des victimes et les polémiques sur la qualification génocidaire
}

Historians' Controversies regarding the French Revolution: the Polemics about the Number of Victims and the Legal Qualification of 'Genocide'

\section{Marc Angenot}

\section{(2) OpenEdition Journals}

\section{Édition électronique}

URL : http://journals.openedition.org/aad/3585

DOI : 10.4000/aad.3585

ISSN : 1565-8961

\section{Éditeur}

Université de Tel-Aviv

\section{Référence électronique}

Marc Angenot, «Querelles historiennes sur la Révolution française : l'argumentation par le chiffre des victimes et les polémiques sur la qualification génocidaire ", Argumentation et Analyse du Discours [En ligne], 23 | 2019, mis en ligne le 18 octobre 2019, consulté le 28 novembre 2019. URL : http:// journals.openedition.org/aad/3585; DOI : 10.4000/aad.3585

Ce document a été généré automatiquement le 28 novembre 2019.

\section{(c) $(1) \subseteq$}

Argumentation \& analyse du discours est mis à disposition selon les termes de la licence Creative Commons Attribution - Pas d'Utilisation Commerciale - Pas de Modification 4.0 International. 


\section{Querelles historiennes sur la Révolution française : l'argumentation par le chiffre des victimes et les polémiques sur la qualification génocidaire}

Historians' Controversies regarding the French Revolution: the Polemics about the Number of Victims and the Legal Qualification of 'Genocide'

\section{Marc Angenot}

1 Le jugement, aussitôt polarisé en deux camps inconciliables, porté sur la Révolution française, dès le Directoire et tout au long du $19^{\mathrm{e}}$ siècle et $\mathrm{du} 20^{\mathrm{e}}$, passe par une querelle interminable qui se réactive à l'occasion, querelle dont l'histoire n'est pas faite. Je veux parler de la polémique séculaire sur le nombre des victimes occasionnées par les événements de 1789 à 1795 (ou 1799). Elle se rapporte à des travaux successifs qui compilent des additions minutieuses de toutes les victimes confondues pour aboutir à un total effrayant. C'est une entreprise à laquelle se livrent d'abord des publicistes anti-jacobins, des républicains de sensibilité "girondine» - plutôt que des monarchistes et contre-révolutionnaires qui, eux, se bornent à l'hyperbole exorcistique face à un événement diabolique. Joseph de Maistre a écrit en 1797 cette phrase fameuse : «Il y a dans la Révolution française un caractère satanique qui la distingue de tout ce qu'on a vu et peut-être de tout ce qu'on verra ».

Mon objectif n'est pas de trancher entre les polémiques ni de faire la critique des faits et des données allégués : j'en serais bien incapable. Mon but est ici de décrire une argumentation récurrente - je veux montrer qu'on peut la qualifier telle - qui porte jugement, une argumentation voulue concluante par des chiffres alignés sans commentaire. Ce sont des compilations de morts anonymes, des additions de victimes, hommes, femmes et enfants, réduits à des colonnes chiffrées, à une somme, estimation «arrondie » ou, le plus souvent, et plus fallacieusement, calcul à l'unité près. C'est le 
degré zéro de la narration historique et de l'argumentation, mais le total obtenu est censé porter jugement et valoir dénonciation. C'est à ce titre que l'étude des additions des victimes de la Révolution relève de la rhétorique argumentative sous une forme sommaire mais efficace : rien de plus froid, dit-on, qu'une addition, mais la somme obtenue est censée par son énormité même inspirer une insurmontable horreur ${ }^{1}$.

Cette argumentation comptable devant les massacres est à mon sens une idée neuve, une démarche nouvelle issue du traumatisme occasionné par le bouleversement révolutionnaire et par la Terreur. Elle engendre une forme contradictoire de pathos qui va devenir un propre de la modernité : un intense sentiment d'horreur refoulé qui ne peut trouver à s'exprimer que par le biais de «froides" additions de morts à jamais anonymes. Je serai anachronique ici : « La mort d'un seul homme, c'est une tragédie. La mort de millions de personnes, c'est de la statistique " aurait dit Staline - propos évidemment apocryphe, mais répété partout dans la mesure où il caractérise l'horreur des totalitarismes du $20^{\mathrm{e}}$ siècle. Le compilateur girondin atterré qui s'abaisse à la "statistique ", de morts sans noms ni visages refoule dans cet effort comptable une indicible horreur qui en pénètre néanmoins la « froideur » apparente.

Lors même que des publicistes "à chaud ", dès le Directoire, et puis des historiens ont eu raison de vouloir regarder en face les massacres de la « Vendée » (en fait toutes les régions de l'Ouest), d'estimer le nombre de victimes, de documenter la volonté de "populicide » qui y a présidé, il faudrait sans doute confronter dans un esprit de « justice » historique ces atrocités à grande échelle en regardant du côté du passé parler de la dévastation systématique du Palatinat par Louvois en 1689 par exemple. Rien de vraiment nouveau dans l'inhumanité par rapport aux massacres de masse de l'Ancien régime ... si ce n'est leur ampleur. Ce qui est nouveau, c'est le chiffrage utilisé comme argument sans réplique et le genre de pathos refoulé qu'il comporte. C'est en quoi le jugement sur la Révolution, sur les sanglantes premières années de la république est le premier cas d'une argumentation laquelle se trouve aussitôt contrée par une résistance "négationniste» avant la lettre, par le refus des historiens de se livrer à d'oiseuses conjectures - paradigme polémique qui traverse les deux siècles de la modernité pour aboutir à la Shoah et aux génocides du $20^{\mathrm{e}}$ siècle.

5 La preuve par l'ampleur des crimes commis au nom d'une croyance a été un raccourci polémique de tous les temps. Voltaire en son temps s'en servait contre le christianisme avec succès : "Vous verrez, fait-il dire au Curé Meslier (Extrait des sentiments de Jean Meslier 1762), que la religion chrétienne a fait périr la moitié du genre humain ». Une brochure anticléricale anonyme paru en 1790, Les crimes du clergé, cumule précisément les évaluations chiffrées par dizaines et centaines de milliers des victimes de l'Église et des guerres de religion qui ont ensanglanté la France depuis les Croisades. Ce sont précisément des pamphlets anti-catholiques qui les premiers ont recours à des listes comptables et des additions de cette sorte; ils fournissent le modèle des historiens anti-jacobins. En 1794, un autre anonyme, républicain, diffuse un Aperçu rapide sur les crimes des prêtres qui se borne à aligner une telle évaluation à grands traits répartie en 15 catégories, parmi elles " $12^{\circ}$. Douze millions d'Américains, assassinés pour la plus grande gloire de Dieu et l'édification de l'église ». Il additionne enfin un grand total : « Résumons : prêtres, chrétiens, vous avez donné la mort à plus de trente-huit millions d'hommes ".

6 L'effort de compilation systématique à partir de données contemporaines et moins spéculatives du nombre des morts est une démarche d'après les années 1789-95. L'abbé 
Barruel, figure fameuse de l'Émigration, dans ses Mémoires pour servir à l'histoire du jacobinisme publiés à Hambourg en 1803-1805, est un des premiers chiffreurs des «crimes révolutionnaires", mais il lance des estimations excessives et vagues. Il dénonce "ces hommes qui encore aujourd'hui se consolent de trois ou quatre cent mille assassinats, de ces millions de victimes que la guerre, la famine, la guillotine, les angoisses révolutionnaires ont coûtées à la France [...], sous prétexte que toutes ces horreurs amèneront enfin un meilleur ordre des choses " (1803, Discours préliminaire, $\mathrm{XI}$ ). Dans son « Discours préliminaire », l'abbé présentait en ces termes le malheur des temps et amorçait l'explication de ses causes :

Sous le nom désastreux de Jacobins, une secte a paru dans les premiers jours de la Révolution Françoise, enseignant que les hommes sont tous égaux et libres. ... Qu'est-ce donc que ces hommes sortis, pour ainsi dire, tout-à-coup des entrailles de la Terre, avec leurs dogmes et leurs foudres, avec tous leurs projets, tous leurs moyens et toute la résolution de leur férocité ? (Vol. I, 6)

7 Après avoir démontré que la Révolution avait été ourdie de bout en bout par les sociétés secrètes illuministes, il concluait: "Tout le mal qu'elle a fait, elle devait le faire; tous ses forfaits et toutes ses atrocités ne sont qu'une suite nécessaire de ses principes et de ses systèmes » (I, xii). La révolution en France n'était qu'un premier essai des Maçons et des Jacobins, révélait l'abbé émigré qui spéculait sombrement sur l'avenir : l'absurdité et la scélératesse des principes se reflétaient simplement dans l'atrocité des moyens et on n'avait encore rien vu. Les additions et les conjectures hâtives et excessives sont au départ l'expression frustre du traumatisme occasionné par des événements meurtriers et, pour les contre-révolutionnaires, des événements sataniquement inexplicables.

Tout le $19^{\mathrm{e}}$ siècle va disputer inlassablement du nombre des victimes de la Révolution, de l'ampleur des massacres qu'elle a occasionnés et que chaque historien depuis Michelet et Lamartine, réévalue, soit en amplifiant soit en pinaillant. Flaubert a montré que la Révolution est devenue avant tout un « sujet de conversation ». Ainsi débattent, à Chavignolles, Bouvard et Pécuchet. Bouvard est plutôt réacteur alors que l'aigre Pécuchet est de tempérament jacobin. Les deux bonshommes tout en se disputant passent, comme il est de règle chez eux, de la crédulité initiale au plus profond scepticisme :

Dans l'esprit de Bouvard, «Montez au ciel, fils de saint Louis», les vierges de Verdun et les culottes en peau humaine étaient indiscutables. Il acceptait les listes de Prudhomme, un million de victimes tout juste. Mais la Loire, rouge de sang depuis Saumur jusqu'à Nantes, dans une longueur de dix-huit lieues, le fit songer. Pécuchet également conçut des doutes, et ils prirent en méfiance les historiens. Ils n'avaient plus, sur les hommes et les faits de cette époque, une seule idée d'aplomb (chap. 4).

Louis-Marie Prudhomme qui sert de référence à Bouvard, dans les conclusions de son Histoire générale et impartiale additionne effectivement tous les chiffres qu'il a compilés ... et arrive non à un chiffre rond comme suggère Flaubert, mais à un total précis, détaillé sur plusieurs pages - beaucoup trop précis, et double de celui que Flaubert a retenu : 2.022.903 morts jusqu'en l'an V (vol. 6, 522). L'Histoire générale et impartiale multiplie les tableaux partiels et récapitule finalement à l'unité près :

RÉCAPITULATION des individus morts par le fait de la Révolution.

Assemblée constituante.....3753.

Assemblée législative.....8044.

Convention nationale.....1026606. 
Au champ de bataille.........800500.

Colonies.................184000.

Total des morts..........2.022.903. catégories les plus conjecturales de Prudhomme, on trouve : - Individus qui se sont suicidés, pendus, noyés ou jetés par les fenêtres par suite de la Terreur; - Femmes mortes par suite de couches prématurées ; - Morts par la famine ; - Individus devenus fous par la révolution. Etc. Ce qui importe au compilateur c'est d'arriver, au bout d'une computation impossible, à un total énorme additionné à l'unité près: chaque victime compte. Faute de moyens et de sources, ces chiffres ne peuvent être qu'une conjecture épouvantée. Il a néanmoins fait effort de précision là où des données pouvaient être trouvées et il tombe juste sur un point bien documenté. Quatre-vingt-six conventionnels ont péri de mort violente. Ils sont 749 au départ. On compte donc quelque $12 \%$ de morts violentes dans l'Assemblée même. En l'an II et III, il n'en siège guère que la moitié - les autres étant morts, ou en prison, ou cachés à la campagne par prudence.

11 Je rencontre un autre exemple détaillé, compilé plus tard: les Mémoires de l'exécuteur des hautes-œuvres est un épais apocryphe publié en 1830. Je me rapporte au «Chapitre supplémentaire 4 » des Mémoires de l'exécuteur des hautes-œuvres, pour servir à l'histoire de Paris pendant le règne de la Terreur, publiés censément par le bourreau Charles-Henri Sanson $^{2}$. Ce livre dénote un effort, rare pour l'époque, de mesure et de sobriété. L'auteur ou les auteurs comptabilisent le Tableau approximatif des victimes de la révolution, à partir du 14 juillet 1789, époque de la prise de la Bastille jusqu'au 27 octobre 1795, fin de la session de la Convention, à savoir six ans, trois mois, treize jours - ce qui donne en synthèse aux pages $335-6$ :

Mis à la lanterne ; - massacrés dans les émeutes populaires ; - tués lors de la guerre

faite aux châteaux. 400

Par suite des guerres civiles du Comtat Venaisin ; - de Lyon ; - de Bordeaux ; - de Marseille ; - de Toulon ; - de Nancy ; - de Caen ; - de Paris aux journées du 14 juillet (prise de la Bastille); - du 10 août (siège du château des Tuileries); - du 13 vendémiaire (guerre de la Convention contre les sections de Paris)......... 32,000

Massacres de septembre dans Paris et hors Paris. 3,400

Morts sous le fer de la guillotine par jugements des tribunaux révolutionnaires ; des commissions militaires, - temporaires, - populaires.. $13,800^{9}$

Extermination des habitants de la Vendée et Chouannerie.......... 180,000

Républicains tués par les Vendéens et les Chouans....... 87,000

Morts par suite de la famine et de la peur......... 7,000

Emigrés morts à l'étranger, ou de mort naturelle ; ou dans les combats, ou fusillés, guillotinés en France........... 14,000

Fusillés, mitraillés, -noyés, - morts dans des incendies, à Lyon, Toulon, Orange, Nantes, Bédouin, etc......... 18,500

Massacres des blancs et des hommes de couleur dans les colonies...... 50,000

Jacobins mis à mort dans le Midi par les réacteurs. 4,600

Ont péri sous la hache révolutionnaire Louis XVI, la Reine, madame Elisabeth, sœur du roi, et le duc d'Orléans, premier prince du sang............ 4

Mort au Temple par suite de mauvais traitements, le Dauphin (Louis XVII) ..........1

Morts aux armées. 290,000

TOTAL................. 600,705

On voit que le compilateur, tout comme faisait Prudhomme, se garde d'arrondir son addition en isolant deux dernières «catégories" unitaires ce qui lui fait «précisément» 600.705. Ce travail de bénédictin morbide témoigne, disais-je, d’un 
effort assez sobre faute d'accès aux archives quand archives il y avait. Je me borne à plusieurs remarques qui montrent, si nécessaire, combien tout pourtant y est douteux : 1. L'addition même n'est pas juste! Le total, inférieur des deux tiers à la compilation de Prudhomme, moins discordant avec les évaluations avancées aujourd'hui, plutôt inférieur à celles-ci, tomberait plutôt à 700.705. - 2. Le compilateur mélange tout, il est vrai, il additionne des victimes de natures et de causes diverses. Tel est précisément son but, répliquerait-on. Il n'était pas interdit de vouloir faire un bilan global en quelque sorte démographique. -3 . Pour la Vendée avec $180.000+87.000$ morts, il surestime un peu si on se rapporte aux travaux actuels qui du reste ne sont pas d'accord ${ }^{3}$. - 4. Pour les « morts de famine ", il conjecture évidemment faute de mieux quoiqu'aboutissant à un chiffre "modeste » sur une question qui a été peu abordée. Bien que les historiens détaillent des situations de famine dans plusieurs départements en l'an III où pauvres, enfants, vieillards meurent en masse, je ne vois pas qu'une estimation chiffrée ait été accomplie de nos jours à nouveaux frais. Mauvaises récoltes, stagnation du commerce, misère paysanne et ouvrière, et puis famine, c'était un phénomène courant dans l'histoire millénaire de la monarchie; de ce point de vue, la révolution ne fait que prolonger et aggraver une situation récurrente. La famine qui sévit pendant l'hiver 1794-1795 est la dernière famine « au sens propre » de l'histoire de France. Il n'y a pas de doute que le dirigisme économique montagnard y est pour beaucoup, mais non pour tout. - 5. Quant aux "morts de peur ", la catégorie est fantaisiste; si pourtant elle devait rassembler les condamnés morts en prison avant exécution, "de peur » ou de misère physiologique et de mauvais traitements, - catégorie, elle, attestée, - le chiffre serait trop bas. -6 . Pour les "Émigrés guillotinés", il est possible que ce chiffre fasse en partie double emploi avec les « Morts sous le fer de la guillotine par jugements des tribunaux révolutionnaires » (v. plus haut). - 7. Pour les "Massacres des blancs et des hommes de couleur dans les colonies $"=50,000$, le compilateur sous-estime assurément. Par ailleurs, les massacres perdurent à Saint-Domingue-Haïti jusqu'à l'indépendance de la république «noire» proclamée le $1^{\text {er }}$ janvier 1804 , et ces massacres se poursuivent entre le royaume noir du Nord, celui de Henri-Christophe, et le gouvernement «mulâtre » de la capitale sous Pétion et puis sous Jean-Pierre Boyer.

De nos jours, l'historien de la Révolution croit prudent de procéder moins globalement et moins conjecturalement en isolant des épisodes sanglants circonscrits et mieux documentés, mais qui font polémique chez les doctes depuis plus de deux siècles. JeanJoël Brégeon est l'auteur d'une monographie Carrier et la Terreur nantaise. Cet épisode qui a servi tout d'abord aux Thermidoriens de preuve de la scélératesse des «terroristes» jacobins témoigne localement, depuis l'an III, «de l'hystérique sarabande» de chiffres contradictoires. Les passions politiques, la haine voire la négation de l'autre, la nécessité d'alimenter un souvenir édifiant pour les générations futures, ont conduit les témoins puis les historiens à affirmer tout et le contraire de tout. Cela dans les deux camps.... Du côté royaliste, les évaluations de la Terreur nantaise sont bien sûr plus effroyables encore. Quelques chiffres : la Biographie portative des contemporains commencée par le fils de Gracchus Babeuf, continuée par Claude Augustin Vieilh de Boisjoslin et al., parle de 15.000 victimes, rien que pour les fusillades. «Plus raisonnables, les historiens de Nantes, Guépin, Lescadieu et Laurant, évaluent entre 8000 et 12.000 les morts de la Terreur nantaise. Dans son Histoire des Girondins, Lamartine reprend cette estimation. Dommage que Crétineau-Joly, souvent sûr et pondéré, se laisse aller à parler de « vingt-trois noyades de 800 à 900 personnes chacune » $(1987: 285)$. La statistique la plus effarante mentionnée par Brégeon revient 
au duc de Rovigo qui dans la Chronique de France aboutit à un total «précis » de 31724 massacrés sur la Loire.

Le chiffre avancé dans les Mémoires de l'exécuteur pour les victimes de la Terreur blanche dans le Midi et le Lyonnais = 4,600 victimes, apparaît en revanche très sous-estimé. Il faut remarquer toutefois que l'anonyme arrête sa compilation en octobre 1795. Or, les assassinats se prolongent durant tout le Directoire jusqu'en 1799, même si l'an 1795 représente un sommet. Des bandes de tueurs comme les Compagnies de Jéhu, formées par des royalistes avides de vengeance conjoints à de simples brigands, parcourent les campagnes ${ }^{4}$. Les victimes paient leur engagement révolutionnaire, soldats, gendarmes, volontaires ou requis, anciens administrateurs jacobins, mais aussi prêtres constitutionnels «comme le curé de Barbentane, jeté dans la Durance pieds et poings liés » (Brunel 2005 : 1025-1026). Le député Louis Fréron, représentant de la Convention dans le Midi avec Barras en 1793 où il s'était distingué par ses violences et ses rapines, est chargé en octobre 1795 de l'enquête. Dans son rapport il se perd en invectives contre les royalistes et en vaines exagérations. La Terreur blanche inquiète les Thermidoriens du centre et « du côté droit » parce qu'ils voient bien qu'elle vise tous les républicains. Les massacres jacobins de l'an II sont beaucoup mieux documentés que ces massacres des "blancs", sournois, sans archives, sans noms ni responsables, combinés de banditisme et de vendettas personnelles, étalés sur plusieurs années. Edgar Quinet signale ce contraste qui fait que les chiffres avancés, quels qu'ils soient, ne tiennent pas parce que tout a été oblitéré : «Aucun nom n'était prononcé ; l'horreur ne s'attachait à aucun individu. La Réaction, œuvre anonyme, était toute-puissante ; ses barbaries, ne remontant à personne, ne se retournèrent jamais contre elle. Bientôt on nia, plus tard on oublia » $(2009: 824)$.

Le $19^{\mathrm{e}}$ siècle et le $20^{\mathrm{e}}$ siècle reprennent les calculs des victimes sur des archives plus abondantes exhumées (si je puis dire) et parviennent à des chiffres différents de ceux attribués à Lombard de Langres - mais, pour plusieurs des catégories ci-dessus, plus élevés! En ce qui concerne entre autres, seules documentées avec les noms, les exécutions capitales. Durant la période d'un an et demi environ qu'on désigne comme la Terreur, plus de 17.000 personnes sont guillotinées en France, dont 16.594 ont été identifiées. Ces chiffres sont ceux établis par Donald Greer dans The Incidence of the Terror during the French Revolution (1935). Ils sont endossés dans Histoire et dictionnaire de la Révolution française, collectif dirigé par J. Taulard (1987 : 1114). Dans ce chiffre global, sont recensées les 2.500 personnes guillotinées à Paris; 1.306 d'entre elles reposent dans la fosse commune du cimetière de Picpus.

Pour les Massacres de septembre (2-7 sept. 1792), événement sanglant également circonscrit, Pierre Caron (1935), a repris des chiffres publiés dans les années 1860. Les archives de la Ville ayant brûlé durant la Commune, elles ne pouvaient être contrevérifiées. En se servant d'autres archives ignorées jusqu'alors, il dresse un bilan qui demeure adopté jusqu'à nos jours. Il estime que le 2 septembre, le nombre de prisonniers incarcérés dans les prisons de Paris se situe entre 2522 et 2535 ; que le nombre d'exécutions sommaires est entre 1225 et 1392, ce qui donne un pourcentage de victimes compris entre 48 et $55 \%$ des prisonniers. Notre compilateur de 1830 avec 3400 victimes pour les massacres de septembre 1792 dans Paris et hors Paris (on a suggéré ici le chiffre de quelque cinq cents) semble surestimer ce bilan - mais ce n'est pas de façon extravagante. 
En 1986, l'économiste René Sédillot a publié Le coût de la Révolution française. Il fait le bilan démographique d'abord, de 1789 à 1815 inclusivement, dont les conséquences pour la population française s'étendent à tout le siècle :

Deux millions de morts : tel semble être, aux seuls dépens de la France, le coût des guerres de la Révolution et de l'Empire. Deux millions de Français, jeunes pour la plupart, tombent sans postérité. Leur disparition, et du même coup celle des Français qui auraient dû être leurs enfants et petits-enfants, pèseront sur toute la démographie du siècle, et l'appauvriront. » Certains historiens soucieux d'épargner la Révolution attribuent en partie l'ampleur de la dénatalité au $19^{\mathrm{e}}$ siècle, à la restriction des naissances qui commence dans la haute aristocratie et s'étend au $18^{\mathrm{e}}$ siècle aux classes urbaines. À partir de 1800, le nombre des naissances est en chute accentuée en France. Quand les conséquences commencent à en apparaître aux yeux de tous, après la défaite de 1870, le sociologue conservateur Frédéric Le Play l'explique par le «régime de désagrégation de la famille issu de la Terreur et du Code civil » : le partage égalitaire des héritages est un encouragement à l'enfant unique.

Pour ce qui est de la Terreur, Sédillot confirme les évaluations des historiens du $19^{\mathrm{e}}$ siècle : « La plupart opinent pour 17000 condamnations à mort sous la Terreur (après octobre 1793), et pour 35000 victimes en tenant compte des décès en prison et des exécutions sommaires » $(1986: 23)$.

Le débat sur le chiffre des morts de la Terreur resurgit avec une grande régularité dans des revues d'histoire pour "grand public", lors même que les données nouvelles n'abondent pas. Ce débat est aussitôt politisé et naguère toute évaluation des victimes de l'an II était réputée une entreprise contre-révolutionnaire et ne paraissait que dans revues de droite. Toutefois, la doxa à l'égard de Robespierre et des Jacobins a viré depuis la fin du $20^{\mathrm{e}}$ siècle à l'hostilité franche. Ainsi dans un article dans L'Histoire, " Robespierre, la révolution et la terreur », encadré dans le $n^{\circ} 177$ :

Le total des victimes serait de l'ordre de 40000 personnes dont 23000 environ exécutées sans jugement.... A Lyon, la Terreur fait quelque 2000 victimes. Avec les noyades ordonnées à Nantes par le député montagnard Jean-Baptiste Carrier, en marge de l'activité des commissions militaires, on est dans le domaine de la Terreur extra-légale : dans ce cas, la dizaine de noyades collectives en Loire semble avoir fait, dans le seul mois de janvier 1794, entre 2000 et 4000 victimes (Lebrun 1994).

Pour la guerre de Vendée, l'évaluation admise de nos jours, hautement imprécise, est celle proposée par Jacques Dupâquier reprise dans le Dictionnaire historique de la Révolution française de Soboul: "Le nombre des morts provoquées directement ou indirectement par les guerres de l'Ouest n'a pu être inférieur à 200 000, ni supérieur à 300000 » (art. «Population»). 'Fourchette' bien trop vaste et insatisfaisante. Au fond, les premières évaluations sous la Monarchie de Juillet se situaient dans ces limites.

21 Des querelles de chiffres, on passe aux polémiques complémentaires - récurrentes depuis deux siècles encore - sur la qualification juridique des massacres de la Guerre de l'Ouest. Éternel retour des mêmes arguments sans progrès notable. En 2017, on assiste à la relance du débat sur le "génocide " vendéen avec une entrevue d'un juriste dans le Point : « "Détruisez la Vendée !" : la thèse du génocide ». Jacques Villemain requalifie les massacres commis en Vendée, qui correspondent, selon lui, à la définition de génocide au regard du droit actuel. 

argumentative. Raisonner, c'est, au plus élémentaire de ses opérations, distinguer en dénommant. Distinguer (« ...aucun rapport entre la Kolyma et les camps nazis ») ou bien identifier ( « ... nous dénonçons le génocide des bébés phoques ») - or rien, dans le langage ni dans le rapport de l'intelligence au monde, n'interdit clairement de créer des distinctions déraisonnables et des amalgames fallacieux. Les controverses, savantes ou publiques, résurgentes et irréconciliables, ne portent souvent que sur la qualification - au sens juridique de ce terme - d'un fait, d'un acte, d'un événement ou d'un individu, d'une entité politique et forment en soi un sujet de recherche prégnant, susceptible d'éclairer l'histoire de l'opinion et de ses polarisations. C'est ce cas particulier, cas très répandu en réalité que j'ai étudié dans mon essai « La rhétorique de la qualification et les controverses d'étiquetage $»^{5}$.

massacres vendéens ont été dénoncés en leur temps par Gracchus Babeuf - qu'on ne saurait qualifier de contre-révolutionnaire - comme ayant été, verbatim, un « système d'extermination générale ", un "système de dépopulation ", un "populicide », c'est son néologisme, dont il accusait les « ambitieux » du Comité de Salut public. Le besoin de néologiser est un indice frappant du sentiment de l'inouï. On sait que le terme juridique de génocide est un néologisme hybride, gréco-latin, formé en 1944 par Raphael Lemkin, professeur de droit américain d'origine juive polonaise, à partir de la racine grecque genos, "naissance ", "genre ", " espèce ", et du suffixe "-cide» qui vient du latin caedere, "tuer ». Le terme est apparu pour la première fois dans son étude Axis Rule in Occupied Europe en 1944 (chapitre IX) pour définir les crimes perpétrés par les nazis à l'encontre des peuples juif, slaves et tsigane durant la Seconde Guerre mondiale, mais aussi les massacres commis par le gouvernement des Jeunes-Turcs de l'Empire ottoman à l'encontre des Arméniens pendant la Première Guerre mondiale, et ceux dont furent victimes les Assyriens en Irak en 1933. La persécution meurtrière des Roms ou «Tsiganes " par les nazis n'a pas été abordée durant le Procès de Nuremberg, il est certain qu'il y avait aussi "génocide» dans la cadre juridique alors établi. La catégorie juridique appliquée à Nuremberg en 1945 sera reprise et appropriée après cette date par les Arméniens, les Ukrainiens, les survivants des peuples décimés d'Amérique et d'Australasie, par les Tutsis rwandais.

La France a multiplié les lois qui imposent à l'historien et à tout un chacun une "qualification » juridique : la Loi Gayssot (1990) promulguée contre les négationnistes de la Shoah a été suivie de la Loi sur le génocide arménien, puis de la Loi Taubira sur le caractère de "crime contre l'humanité » de la traite négrière et de l'esclavage. En revanche, les historiens français n'appliquent pas volontiers à leur passé colonial la catégorie de "génocide" que les historiens ci-devant "indigènes" appliquent au massacre de Sétif et Setna de mai 1945 avec ses 40.000 morts et aux massacres commis par l'armée française à Madagascar en 1947 avec « peut-être » 100.000 victimes.

La qualification de "génocide » vendéen est anachronique, protestent quelques juristes et en dépit de l'ampleur et l'horreur des massacres, elle est abusive: on ne peut démontrer un plan cohérent d'extermination d'un groupe "ethnique ", plan qui aurait été appliqué à la lettre - lors même que les morts atteignent le chiffre de 22 à $25 \%$ des populations touchées; $50 \%$ dans les départements de la Vendée militaire. Tel général républicain se conduit en boucher, tel autre montre un degré de modération. Objection faible. L'idée que les crimes n'existent pas avant d'être nommées est une forme de nominalisme sophistique. - À ce compte, il n'y aurait pas eu de crime contre l'Humanité

Argumentation et Analyse du Discours, 23 | 2019 
avant Nuremberg! Pour Jean-Joël Brégeon, historien de la Terreur nantaise, "manifestement», les Vendéens formaient un groupe original passible d'un " génocide»: « ruraux, recourant à des variantes patoisantes de l'idiome national, catholiques fervents, les "brigands" tranchaient par leur physionomie et leurs pratiques culturelles sur les habitants des villes, plus cosmopolites ; plus affinés ou, en tout cas, différents dans leur façon d'être » (1987: 279). L'idée que les insurgés de l'Ouest aient pu constituer une catégorie de sous-hommes incapables d'accéder au nouveau dogme égalitaire, ajoute Brégeon citant des sources abondantes, semble avoir été fort répandue dans le camp républicain. Les conflits déclenchés par la Révolution française ont quelque chose de neuf, de «moderne » dans l'histoire de la violence de masse, ils marquent un seuil qualitatif : la conscription, la levée en masse précisément, la «guerre totale " - locution qui se rencontre une première fois dans un discours de Robespierre (Lafon 2013). Le «populicide» de l'Ouest de la France est documenté de première main par les rapports enthousiastes des généraux de la République. Il suffit de les citer. Lettre au Comité de Salut Public:

Il n'y a plus de Vendée! Elle est morte sous notre sabre libre, avec ses femmes et ses enfants. Je viens de l'enterrer dans les marais et dans les bois de Savenay. J'ai écrasé les enfants sous les pieds de mes chevaux, massacré les femmes qui n'enfanteront plus de brigands. Je n'ai pas un prisonnier à me reprocher. J'ai tout exterminé. Nous ne faisons pas de prisonniers, car il faudrait leur donner le pain de la liberté, et la pitié n'est pas révolutionnaire.... Les routes sont semées de cadavres. Il y en a tant que sur plusieurs points ils font des pyramides (Gérard 1993 : 253).

Ainsi le général François Westermann, dantoniste et "indulgent » qui sera à ce titre bientôt guillotiné, rend-il compte à Paris de ses hauts faits, conformes d'ailleurs aux instructions de la Convention qui, par décret du 2 août 1793, "avait ordonné la destruction systématique et l'incendie de tout le pays, récoltes comprises, en même temps que la mise à mort des rebelles ». Ainsi épilogue René Sédillot qui cite plusieurs autres rapports analogues (1986:24).

À partir du cas d'origine que je viens de décrire, il faudrait faire l'histoire des computations, toutes disputées, ultérieures à travers guerres, massacres, génocides et caractériser les usages juridico-politiques des chiffres de "pertes humaines ». Le monde pendant le siècle de 1815-1914, le monde d'après le Congrès de Vienne et jusqu'à l'ainsi nommée Grande Guerre, ce monde en marche vers le Siècle des génocides (Bruneteau 2004) connaît d'innombrables guerres continentales et de nombreux massacres «coloniaux " culminant avec trois génocides caractérisés, celui des Cherokees, 1828 (McCurdy 2019 : 37), des Tasmaniens, entièrement accompli vers 1876, des Hereros perpétré dans le Deutsch-Südwestafrika, actuelle Namibie, en 1904. Le génocide des Tasmaniens par les colons anglais - l'un des seuls ayant été jusqu'au bout de sa solution finale avec quelques autres éliminations de «tribus " de moindre ampleur dans l'histoire coloniale - s'étale sur un court demi-siècle. Truganini née vers 1812 et décédée le 7 mai 1876, est une femme dont on exhibe la photo considérée comme la dernière des Aborigènes de Tasmanie. Après son enterrement, son corps est exhumé et son squelette suspendu à titre de curiosité dans une vitrine du Tasmanian Museum où il resta jusqu'en 1947. Il y a peu de livres sur les génocides intégraux : nul Front de libération ne subsiste pour revendiquer leur mémoire. 
Ce sont les pacifistes d'autrefois qui se livrent aux calculs minutieux des "pertes humaines " occasionnées par les guerres entre pays "civilisés", - et ce sont des économistes aussi qui déplorent le "gaspillage " tant financier qu'humain. Ainsi de Paul Leroy-Beaulieu (1843-1916), économiste libéral qui compile Les guerres contemporaines 1853-1866 (1868). Il prétend établir les chiffres des pertes matérielles, «tant en argent qu'en hommes », des guerres qui ont affligé l'humanité ["civilisée » dis-je] en l'espace de quatorze ans - de la Guerre de Crimée à la Guerre austroprussienne de 1866 en passant par la Guerre de sécession, Leroy-Beaulieu, qui ne s'est guère informé sur les massacres de la Guerre du Paraguay (" de la Triple Alliance ») et en sous-estime les victimes, qui ne s'occupe pas de la Chine ${ }^{6}$ ni de l'Orient et fort peu des entreprises coloniales, aboutit néanmoins à un total de 1.743.491 hommes enlevés par la guerre aux «peuples civilisés». Ces massacres ont coûté aux États 47 milliards 830 millions de francs-or. Ce petit livre paraît deux ans avant la Guerre francoprussienne et avant la grande expansion coloniale de la fin du siècle.

Je ne puis dans l'espace qui m'est dévolu mentionner les étapes et les aspects politiques d'un phénomène hérissé de controverses et qui est de plus en plus délicat à traiter, alors que les crimes de masse se rapprochent de nous, et alors que se déchaînent à chaque fois des querelles chiffrées et des dénégations sophistiques qui mériteraient une étude diachronique et comparative. On aboutirait aux violentes polémiques contemporaines sur les additions confrontées des victimes des totalitarismes du $20^{\mathrm{e}}$ siècle.

Je n'ai nul besoin de rappeler l'odieuse question des négationnistes de la Shoah sur lesquels les livres abondent ${ }^{7}$. Arthur Butz, professeur d'électrotechnique à l'Université Northwestern, dans The Hoax of the Twentieth Century (2003), et Richard Harwood, dans Did Six Million Really Die? (1978), « démontrent » que l'Holocauste n'a jamais eu lieu ou a été immensément " exagéré »; ils offrent comme tous leurs congénères une explication conspiratoire : l'Holocauste est un mensonge ourdi par les Sionistes pour atteindre leur éternel plan de domination mondiale et pervertir les esprits des Gentils. On doit reconnaître toutefois que le révisionnisme, à savoir la tendance idéologiquement motivée sinon à nier du moins à sous-estimer énormément l'ampleur de certains massacres est un phénomène bien plus répandu que le «cas" immonde des négationnistes de la Shoah. Il reste à produire une histoire européenne et américaine des négationnismes d'État, échappatoire idéologique toujours renaissante.

Les controverses franco-françaises récurrentes depuis trente-cinq ans sur la «responsabilité » des idées léninistes, de la «morale» et de la vision du monde bolchevik dans le Malheur du siècle remontent à la publication de L'archipel du Goulag en 1974 et à sa vaste diffusion dans le monde (l'auteur russe ne disait rien qui ne fût déjà connu à travers les livres de Victor Serge, Panaït Istrati, Boris Souvarine, Viktor Kravchenko, mais une censure énorme s'est peu à peu levée à cette époque). Les tentatives brutales du PCF pour étouffer l'impact de Soljénitsyne ont l'effet contraire, le succès de librairie est immense; la critique de l'URSS se libère des réticences et «nuances » qu'on y mettait naguère - le chantage à l'anti-communisme, imparable depuis 1945, a cessé d'opérer à plein. En 1975-76, le pionnier de l'anti-totalitarisme, Claude Lefort publie Un homme en trop, André Glucksmann, ci-devant gauchiste flamboyant, La cuisinière et le mangeur d'hommes, Jean-François Revel, La tentation totalitaire. Ces controverses culminent dans les années de la fin du siècle sur la « mise en accusation» globale de l'Idée par un groupe d'historiens du communisme qui 
prétendent l'observer sous toutes ses formes, dans tous ses régimes et sur la planète entière - mise en accusation accueillie par les "hauts cris", littéralement, des défenseurs des "Idées généreuses" et de leur immunité face à l'histoire. La controverse historienne la plus violente de la fin $\mathrm{du} 20^{\mathrm{e}}$ siècle est dès lors celle sur les victimes des régimes communistes. Elle prolonge le présent essai sur 1789-1795, car elle montre une nouvelle fois le heurt des partisans de comptabilités macabres avec les réticents, les sceptiques et les négationnistes par conviction militante. Dans le contexte de l'Atroce $20^{\mathrm{e}}$ siècle, ce sont en effet les victimes des régimes communistes, longtemps occultées par les victimes du nazisme et des fascismes, qui ont réclamé tardivement « l'attention » historienne, attention à la fois légitime et politiquement motivée. La fin $\mathrm{du} 20^{\mathrm{e}}$ siècle a vu se développer ces nouvelles querelles de chiffres. L'addition de tous les morts du communisme, formant un grand total de 96 millions de victimes pour tous les régimes qui se sont réclamé de cette idéologie au cours du siècle, a pu paraître une manière de procéder plus polémique que sensée. Elle a été reprochée à l'«Introduction» de Stéphane Courtois au Livre noir du communisme (1997 : 20). Elle pouvait, à son sentiment, se justifier en raison du fait que tous les régimes de ce type étaient coulés dans le même moule et sont des variantes de la même conception idéocratique du pouvoir. "Les régimes communistes ont commis des crimes concernant environ 100 millions de personnes contre environ 25 millions de personnes au nazisme ", contraste en "arrondissant" au passage Courtois dont l'intention explicite est de montrer le communisme comme le pire, le plus sanguinaire de tous les totalitarismes. Martin Malia, auteur de The Soviet Tragedy, a commenté le choc occasionné dans le public volontiers amnésique par ce chiffrage :

Certes, chacun des épisodes majeurs de la tragédie - le goulag de Staline, le Grand Bond en avant et la Révolution culturelle de Mao Zedong, les Khmers rouges de Pol Pot - a eu son triste moment de notoriété. Mais ces horreurs se sont bientôt évanouies dans l'« histoire » et personne ne s'est avisé d'en additionner le total et de le proposer au public. L'extraordinaire importance de ce total, du coup, explique en partie le choc provoqué par le livre. Cependant, le vrai choc est venu de l'inévitable comparaison de ce total avec celui du nazisme qui, avec une évaluation de vingt-cinq millions de victimes, apparait nettement moins meurtrier que le communisme (Malia 2002).

Il subsiste néanmoins, au degré extrême d'inhumanité où nous sommes parvenus, une différence que l'on jugera décisive ou non - je ne crois pas que l'on puisse raisonnablement trancher ce point. Elle met les génocides ourdis et accomplis par le national-socialisme, inspirés par un millénarisme pervers, la Shoah et le génocide des Roms, dans une catégorie suprême. Comme l'écrit Richard Evans: "There was no Soviet Treblinka, built to murder people on their arrival ${ }^{8}$. "

Les victimes du régime soviétique dont ni les Russes en leur majorité ni certains Occidentaux « de gauche " ne souhaitent se souvenir, demeurent en toute rigueur incalculables et sont aussi à jamais anonymes. Il n'y a guère que pour la Shoah que le Yad Vashem a pu pieusement retrouver et publier en ligne les noms d'un peu plus de quatre millions et demi d'entre les victimes'. 


\section{BIBLIOGRAPHIE}

anon. 1794. Aperçu rapide sur les crimes des prêtres, ou Relevé exact des assassinats commis au nom de la religion depuis la naissance du christianisme jusqu'à nos jours (Les archives de la Révolution française, en ligne, 8.1722)

Babeuf, Gracchus et al. 1836. Biographie universelle et portative des contemporains (Paris: Chez l'éditeur) [commencée en 1825 par le fils de Babeuf et continuée par Claude Augustin Vieilh de Boisjoslin et al.]

Bacot, Paul, Dominique Desmarchelier \& Sylvianne Rémi-Giraud (éds). 2012. « Chiffres et nombres dans l'argumentation politique $»=$ Mots 100

Barruel (abbé). 1803. Mémoires pour servir à l'histoire du jacobinisme (Hambourg : Fauché)

Brégeon, Jean-Joël. 1987. Carrier et la Terreur nantaise (Paris : Perrin)

Bridonneau, Pierre. 1997. Oui, il faut parler des négationnistes : Rogues, Faurisson, Garaudy et les autres (Paris : Le Cerf)

Brunel, Françoise. 2005. «Terreur blanche », Albert Soboul, Dictionnaire historique de la Révolution française (Paris : Quadrige)

Bruneteau, Bernard. 2004. Le siècle des génocides (Paris : Colin)

Butz, Arthur. 2002. The Hoax of the Twentieth Century (Carshalton : Historical Review Press)

Caron, Pierre. 1935. Les Massacres de Septembre (Paris : La Maison du Livre français)

Courtois, Stéphane, Nicolas Werth, Jean-Louis Panné, Andrzej Paczkowski, Karel Bartosek, JeanLouis Margolin. 1997. Le livre noir du communisme. Crimes, terreur, répression (Paris : Laffont)

Courtois, Stéphane éd. 2002. Du passé faisons table rase! Histoire et mémoire du communisme en Europe (Paris : Laffont)

Dupâquier, Jacques. 1989. Art. « Population/Démographie ", Soboul, Albert. 1989. Dictionnaire historique de la Révolution française. Jean-René Suratteau et François Gendron dir. (Paris : PUF)

Finkielkraut, Alain. 1982. L'avenir d’une négation, réflexion sur la question du génocide (Paris : Seuil)

Finkielkraut, Alain. 1989. La mémoire vaine: du crime contre l'humanité (Paris : Gallimard)

Gérard, Alain. 1992. La Vendée : 1789-1793 (Seyssel : Champ Vallon)

Godin, Christian. 2000. Négationnisme et totalitarisme (Nantes : Pleins Feux)

Glucksmann, André. 1976. La cuisinière et le mangeur d'hommes (Paris : Seuil)

Greer, Donald. 1935. The Incidence of the Terror during the French Revolution: A Statistical Interpretation (Cambridge MA, Harvard U. P.)

Igounet, Valérie. 2000. Histoire du négationnisme en France (Paris : Seuil)

Katz, Steven T. 2019. Holocaust Studies : Critical Reflections (London : Routledge)

Koren, Roselyne. 2003. « Contribution à l'étude des enjeux de la rhétorique laconique : le cas des indications chiffrées », Topique 2, 111-124

Koren, Roselyne. 2009. "Le récit de chiffres : enjeux argumentatifs de la "narrativisation" des chiffres dans un corpus de presse écrite contemporain ", A contrario 2, 66-84 
Lafon, Jean-Marc. 2013. Guerres et conflits dans le monde au XIXe siècle 1792-1914 (Paris : Ellipses)

Lefort, Claude.1975. Un homme de trop (Paris : Belin)

Lebrun, François. 1994. « Robespierre, la révolution et la terreur », L'Histoire 177

Leroy-Beaulieu, Paul. 1868. Les guerres contemporaines 1853-1866 (Paris : Pichon-Lamy)

Lemkin, Raphael. 1944. Axis Rule In Occupied Europe: Laws Of Occupation, Analysis Of Government, Proposals For Redress (Washington DC: Carnegie Endowment for International Peace, Division of International Law). Rééd. 2012 (Lexington Books)

Malia, Martin. 2002. « Usages de l'atrocité ", S. Courtois éd. Du passé faisons table rase! Histoire et mémoire du communisme en Europe (Paris : Laffont), 211-226

McCurdy, John Guilbert. 2019. Quarters: The Accommodation of the British Army and the Coming of the American Revolution (New York : Cornell U.P.)

Michel, Natacha éd. 1997. Paroles à la bouche du présent: le négationnisme, histoire ou politique? (Marseille : Dante)

Prudhomme, Louis-Marie. 1797. Histoire générale et impartiale des erreurs, des fautes et des crimes commis pendant la Révolution française au Vol. VI, parue en l'an V (hiver 1797)

Quinet, Edgar. 2009 [1865]. La Révolution, préf. de Claude Lefort (Paris : Belin Poche)

Revel, Jean-François. 1976. La tentation totalitaire (Paris : Le Livre de poche)

Samson, Charles-Henri. 1830. Mémoires de l'exécuteur des hautes-œuvres, pour servir à l'histoire de Paris pendant le règne de la Terreur (Paris : Chez les principaux libraires)

Sédillot, René. 1968. Le coût de la Révolution française (Paris : Perrin)

Tulard, Jean, Jean-François Fayard \& Alfred Fierro. 1987. Histoire et dictionnaire de la Révolution française, 1789-1799 (Paris : Laffont Bouquins)

Vidal-Naquet, Pierre. 1987. Les assassins de la mémoire (Paris : La Découverte)

Yad Vachem (Base de données) http://yvng.yadvashem.org/index.html?language=fr

\section{NOTES}

1. La revue Mots a sorti un numéro $(100,2012)$ qui porte sur la question abordée ici, "Chiffres et nombres dans l'argumentation politique ", sous la direction de Paul Bacot et al. Je signale aussi particulièrement l'essai de Roselyne Koren, « Contribution à l'étude des enjeux de la rhétorique laconique » (2003) et son article : « Le récit de chiffres » (2009). Je m'inspire ici des suggestions de méthode rencontrées dans ces articles.

2. Éditeur et auteur probable : Vincent Lombard de Langres (1765 ? -1830).

3. Je parle de ces controverses plus loin.

4. Joseph Fouché, dans ses Mémoires (1824:25), confronte Terreur jacobine et Terreur blanche : «L'écroulement subit du régime affreux qui tenait toute la nation entre la vie et la mort fut sans doute une grande époque d'affranchissement ; mais [...] on passa de la terreur à l'anarchie, de l'anarchie aux réactions et aux vengeances. La révolution fut flétrie dans ses principes et dans son but ; les patriotes restèrent exposés long-temps à la rage des sicaires organisés en compagnies du Soleil et de Jésus » (1025-1026).

5. Repris dans le dernier cahier de Discours social, «Interventions critiques VI » = vol. 47, paru en 2016. 
6. En Chine au milieu du $19^{\mathrm{e}}$ siècle, la Guerre des T'ai Ping fait plus de vingt millions de morts, certains historiens chinois disent trente. T'ai Ping, nom chinois du Royaume céleste de la Grande Paix, est le nom d'une puissante rébellion qui secoue à partir de 1851 le centre et le sud de la Chine. Le fondateur du mouvement, Hong Xiuquan, se dit le « frère cadet de Jésus ».

7. Comme ouvrages exemplaires je cite Vidal-Naquet 1987, Finkielkraut 1982 et 1989, Igounet 2000, Bridonneau1997, Michel1997, Godin 2000 (v. la bibliographie).

8. R. Evans ibid., 122, Cité d'après Katz 2019.

9. Site de Yad Vachem, Jérusalem : «Four and a half of the six million Jews murdered by the Nazis and their accomplices are commemorated here. This database includes information regarding victims of the Shoah » http://yvng.yadvashem.org/index.html?language=fr

\section{RÉSUMÉS}

À partir du Directoire et à travers les $19^{\mathrm{e}}$ et $20^{\mathrm{e}}$ siècles des polémiques récurrentes se sont développées sur le nombre des victimes de la Révolution. Cette argumentation statistique devant les massacres de masse - évidemment très conjecturale - représente une démarche nouvelle issue du traumatisme occasionné par le bouleversement révolutionnaire et la Terreur. Elle engendre une forme contradictoire de pathos qui va devenir un propre de la modernité : un intense sentiment d'horreur refoulé qui ne trouve à s'exprimer que par le biais de "froides " additions de morts anonymes. Quant aux événements de la Vendée, ils suscitent dès 1795 la qualification néologique de "populicide » qui réapparaît en 1945 sous la forme "génocide ». De la Révolution aux guerres des deux siècles modernes, aux conquêtes coloniales et aux massacres de populations entières, historiens, démographes et économistes mais aussi les pacifistes ont repris jusqu'à nous la tâche de chiffrer les morts et d'en tirer argument.

Starting with the Directoire (1795) and continuing throughout the $19^{\text {th }}$ and $20^{\text {th }}$ centuries, endless polemics broke over the number of victims massacred during the French Revolution. Though highly speculative, the use of statistics as a means of argumentation represents a new discursive phenomenon triggered by the trauma of revolutionary upheaval and the reign of Terror. Paradoxically, statistics gave rise to a new kind of pathos which was to become a characteristic of Modernity: An intense feeling of repressed horror was conveyed through the apparent matter-of-fact stockpiling of the number of anonymous victims. As early as 1795 during the War in the Vendée which had its own share of massacres, a neologism was coined: "Populicide." It will reappear in 1944 under the term "Genocide". From the French Revolution on -- and through the wars, colonial conquests, and ethnic massacres over the two centuries of Modernity up to the present day - historians, demographers, economists and pacifists have taken up again and again the task of evaluating the number of victims and using the "total" as a decisive argument.

\section{INDEX}

Mots-clés : génocide, nombre des victimes, polémiques, qualification légale, Révolution française

Keywords : French Revolution, genocide, legal qualification, polemics, number of victims 
AUTEUR

MARC ANGENOT

Université McGill, Montréal 